\title{
Tuning FORM with large calculations
}

\author{
J.A.M. Vermaseren ${ }^{\mathrm{a}}$ \\ ${ }^{a}$ NIKHEF, P.O. Box 41882, \\ 1009 DB, Amsterdam
}

Some recent additions to FORM are discussed. In particular large file support and the tablebases are presented.

\section{Introduction}

Traditionally FORM [ [1] is called a program for particle theory. This is however a misconception that follows from a desire of putting labels on things. FORM is a program for many fields of science in which large formulae occur, like in deep perturbative expansions. Its dealing with non-commutative objects makes it also very suitable for mathematics calculations [ 2, 3. And it has also been used successfully in the field of Euler-Zagier sums [ [4 in which the results of certain categories of sums can only be obtained by solving large sets of equations. Of course FORM has been mostly tested in perturbative quantum field theory. However its speed and the potential size of its expressions should make FORM very attractive for many scientists.

New features are always looked at from a more generic viewpoint. This makes them useful for as many people as possible. Some of these new features are:

- \$-variables which allow a high level of control over the organisation of a program. Version 1 and 2 of FORM never had this flexibility.

- Write to file facilities. This allows even dynamical addition to running programs.

- Large file support. Now also 32-bits processors can deal with intermediate expressions and files of more than 2 Gbytes, provided the operating system supports this (as in the ext-3 file-system versions of Linux).

- Better support for large tables.

- The tablebase. This is a database-like facility for extremely large tables. It was in- spired by a calculation [5, 6] in which there were more than 20000 table elements, each of which occupied on average more than 20 Kbytes. To compile all these table elements in each program that might need some of the elements would be wasteful and slow, even in FORM. Now there are facilities by which the program can determine what is needed and when, and only those elements will be compiled at the proper moment.

\section{Some examples}

The first example concerns a run which is much like a benchmark used originally by D. Fliegner [ 7] to test the parallel version of FORM. Later this test was taken over by R. Kreckel [8] to compare the $\mathrm{GiNaC}$ system with other symbolic systems. Here we have modified it somewhat to allow the intermediate expression to surpass 4 Gbytest.

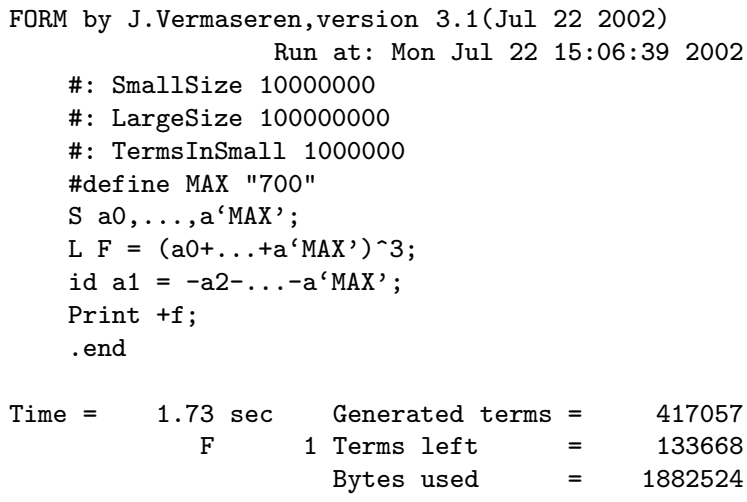

${ }^{1}$ A direct extension of the original test in which the power is 2 would run into the limit of 6000 variables (on 32-bit systems) before the 4 Gbyte limit would be reached 


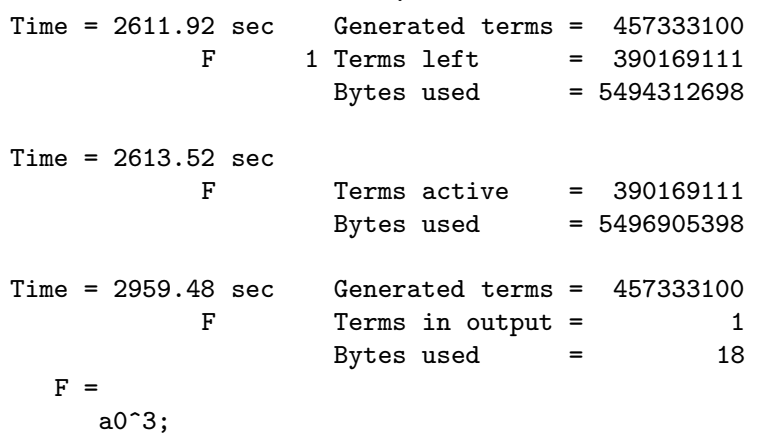

The run was on a notebook computer with a $850 \mathrm{MHz}$ Pentium, 500 Mbytes memory and RedHat 7.3 Linux.

The next example shows the dynamic extension of tables during a run. It uses the $\$$-variables and the resulting table elements are also appended to a file. This way each new run can start by reading all results of the previous jobs. This mechanism was used to run and tabulate more than 20000 integrals in the computation of basic building blocks for the three loop structure functions in deep inelastic scattering. Sometimes more than 1000 integrals were done in a single (rather lengthy) run.

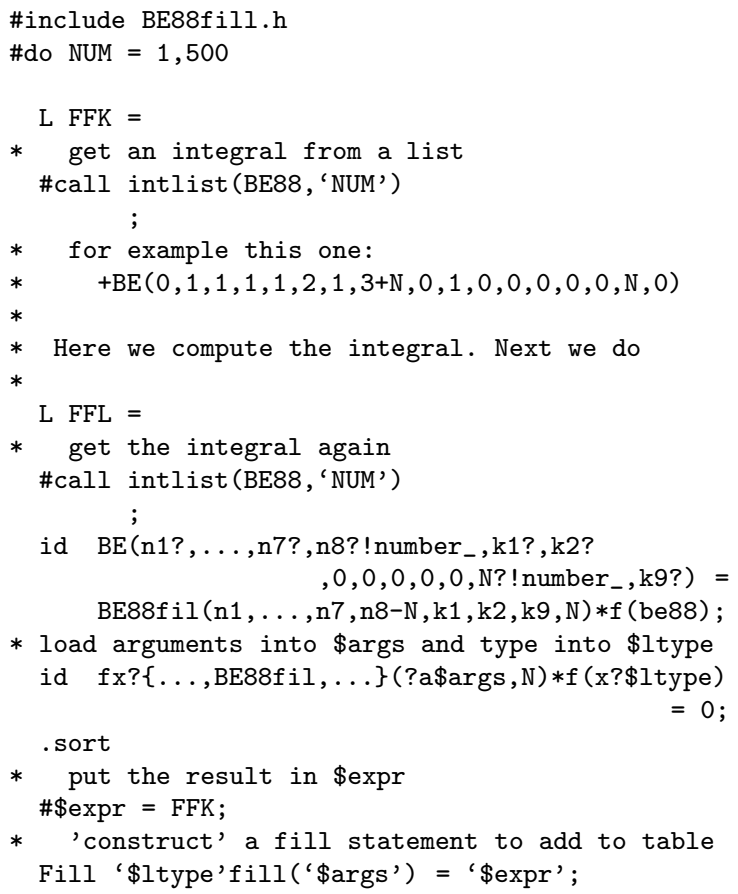

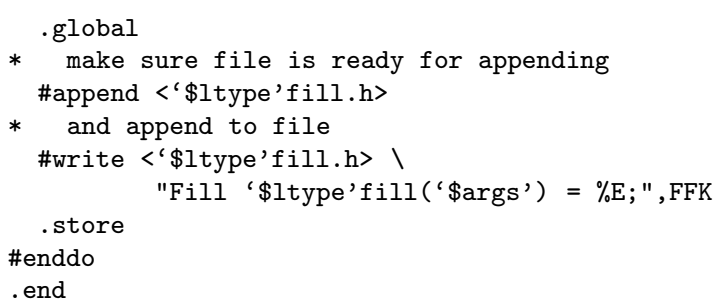

\section{The tablebase}

Faced with hundreds of megabytes of table elements of which we may typically need only a few in each job (but we cannot say in advance which) we need a special database structure. We want a database for FORM with the features:

- FORM reads at first only an index of the database.

- At a specified time FORM can determine which elements are actually needed.

- At a specified time FORM will load and compile these elements.

- When the user speciefies it, the elements will be used.

- The elements can be stored in gzipped [9] form (saves a factor 4).

Of course such 'tablebases' need a number of control commands amoung which should be commands for

- Creating a new tablebase.

- Adding tables and table elements to the tablebase.

- Investigating what is in the tablebase.

- Removing elements from the tablebase.

- Cleaning up a tablebase.

- Loading the index and compiling 'stubbs'.

- Loading and compiling individual elements.

- Loading and compiling complete tables.

- Loading and compiling indicated elements. 
- $\cdots$ and probably more $\cdots$.

The stubbs are intermediate expressions. They replace an object by an indicator that this table element exists in the tablebase. The advantage of this is that the object does not need to be manipulated by other routines that would deal with cases that are not in the tablebase, but yet we do not replace it by potentially lots of terms until we are ready for manipulating those terms. Let us see how this works out.

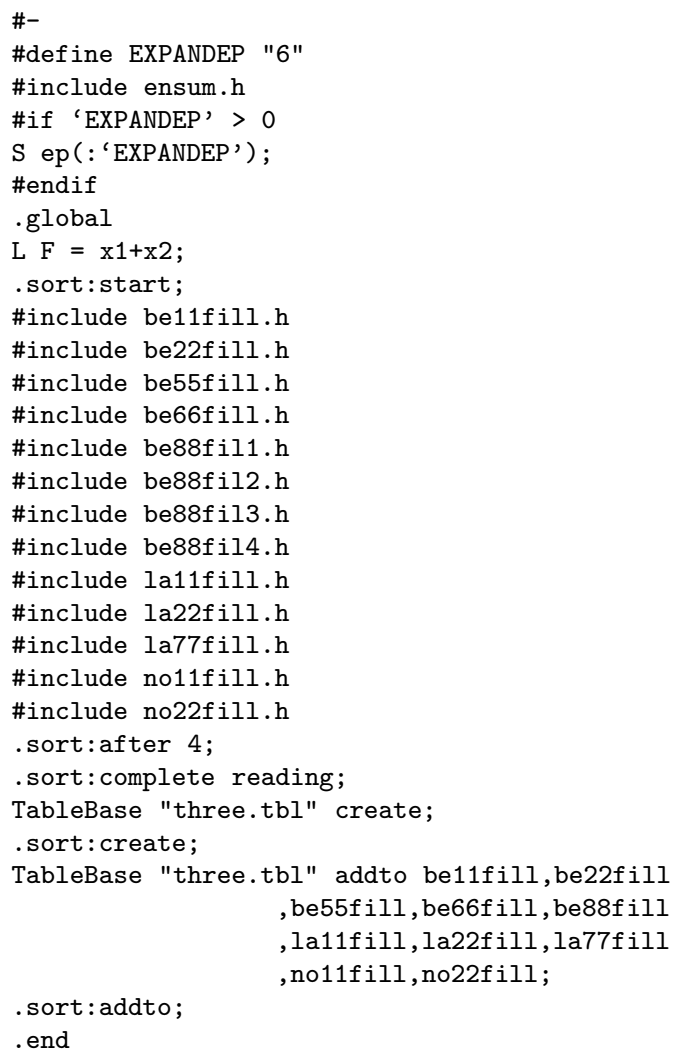

This program gives the output

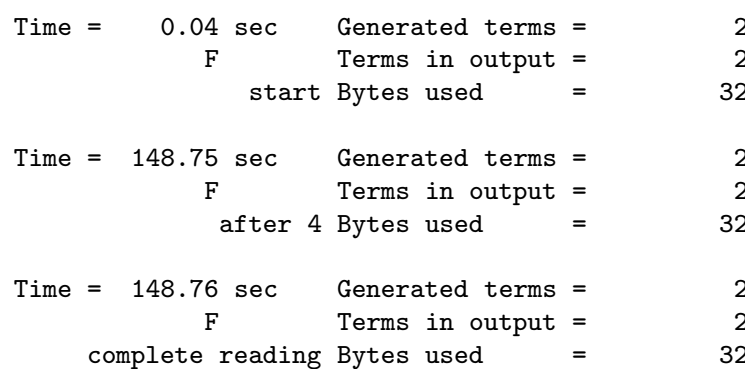

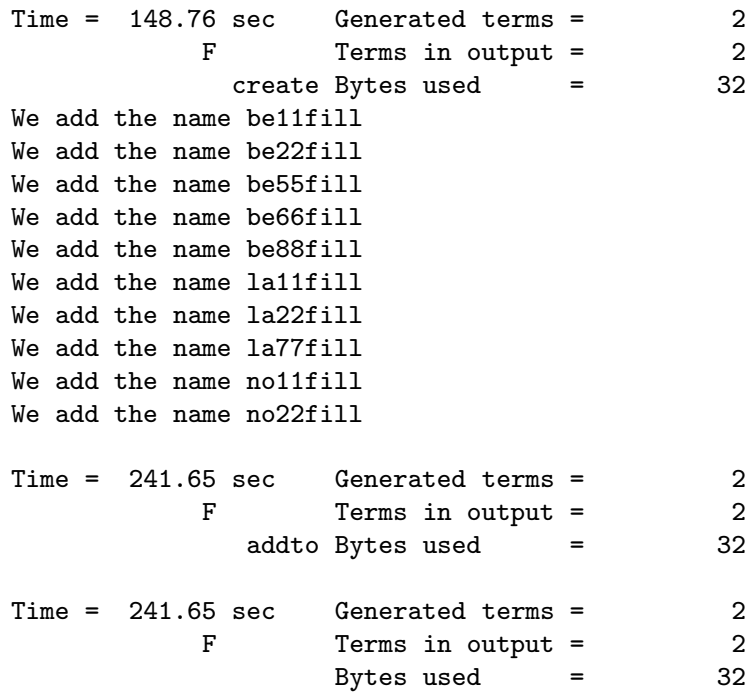

The running times refer to a Pentium 850. The first part shows the reading and compilation of the entire tables. The second part is the compression and the writing into the tablebase. How big are these files?

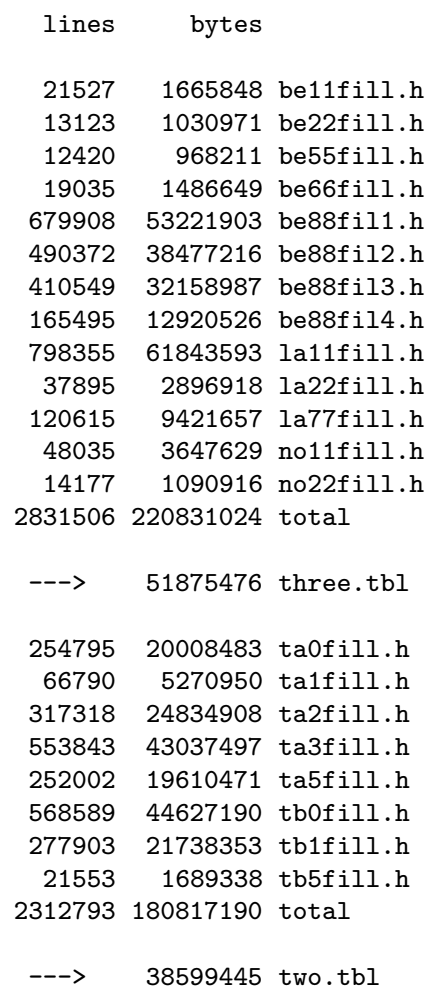




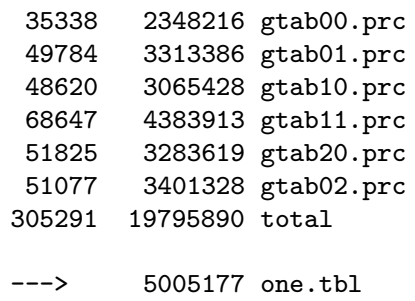

These are the three loop, two loop and one loop tabulated integrals respectively.

There is already one pleasant spinoff. When we just load this one file three.tbl and enter and compile all elements we have

\begin{tabular}{|c|c|c|c|c|}
\hline Time $=$ & $\begin{array}{r}0.07 \\
F\end{array}$ & $\begin{array}{l}\text { sec } \\
\text { start }\end{array}$ & $\begin{array}{l}\text { Generated terms } \\
\text { Terms in output } \\
\text { Bytes used }\end{array}$ & $\begin{array}{l}= \\
= \\
=\end{array}$ \\
\hline Time $=$ & $\begin{array}{r}0.20 \\
F\end{array}$ & $\begin{array}{l}\text { sec } \\
\text { open }\end{array}$ & $\begin{array}{l}\text { Generated terms } \\
\text { Terms in output } \\
\text { Bytes used }\end{array}$ & $\begin{array}{l}= \\
= \\
=\end{array}$ \\
\hline Time $=$ & $\begin{array}{r}0.59 \\
F\end{array}$ & $\begin{array}{l}\text { sec } \\
\text { load }\end{array}$ & $\begin{array}{l}\text { Generated terms } \\
\text { Terms in output } \\
\text { Bytes used }\end{array}$ & $\begin{array}{l}= \\
= \\
=\end{array}$ \\
\hline Time $=$ & $\begin{array}{r}124.80 \\
F\end{array}$ & $\begin{array}{l}\text { sec } \\
\text { enter }\end{array}$ & $\begin{array}{l}\text { Generated terms } \\
\text { Terms in output } \\
\text { Bytes used }\end{array}$ & $\begin{array}{l}= \\
= \\
=\end{array}$ \\
\hline Time $=$ & $\begin{array}{r}124.80 \\
F\end{array}$ & $\mathrm{sec}$ & $\begin{array}{l}\text { Generated terms } \\
\text { Terms in output } \\
\text { Bytes used }\end{array}$ & $\begin{array}{l}= \\
= \\
=\end{array}$ \\
\hline
\end{tabular}

and we see that the fact that the file is compressed saves much time on the reading. Loading alone, the process of reading the index and compiling a complete list of 'stubbs' takes about 0.4 sec. which indicates that we have eliminated the whole problem of slow startup.

Let us now try to use this.

$\#-$

.global

$\mathrm{L} F=\operatorname{LA}(1, \mathrm{~N}+1,1,1,1,1,1,1,0, \mathrm{~N}, 0,0,0,0,0,0,3)$ $+\mathrm{LA}(1, \mathrm{~N}+1,1,8,1,1,1,1,0, \mathrm{~N}, 0,0,0,0,0,0,3)$;

Print $+\mathrm{f}+\mathrm{s}$;

. sort

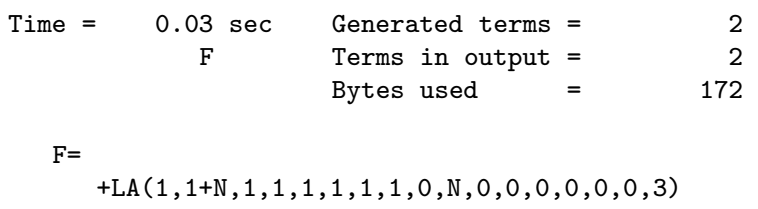

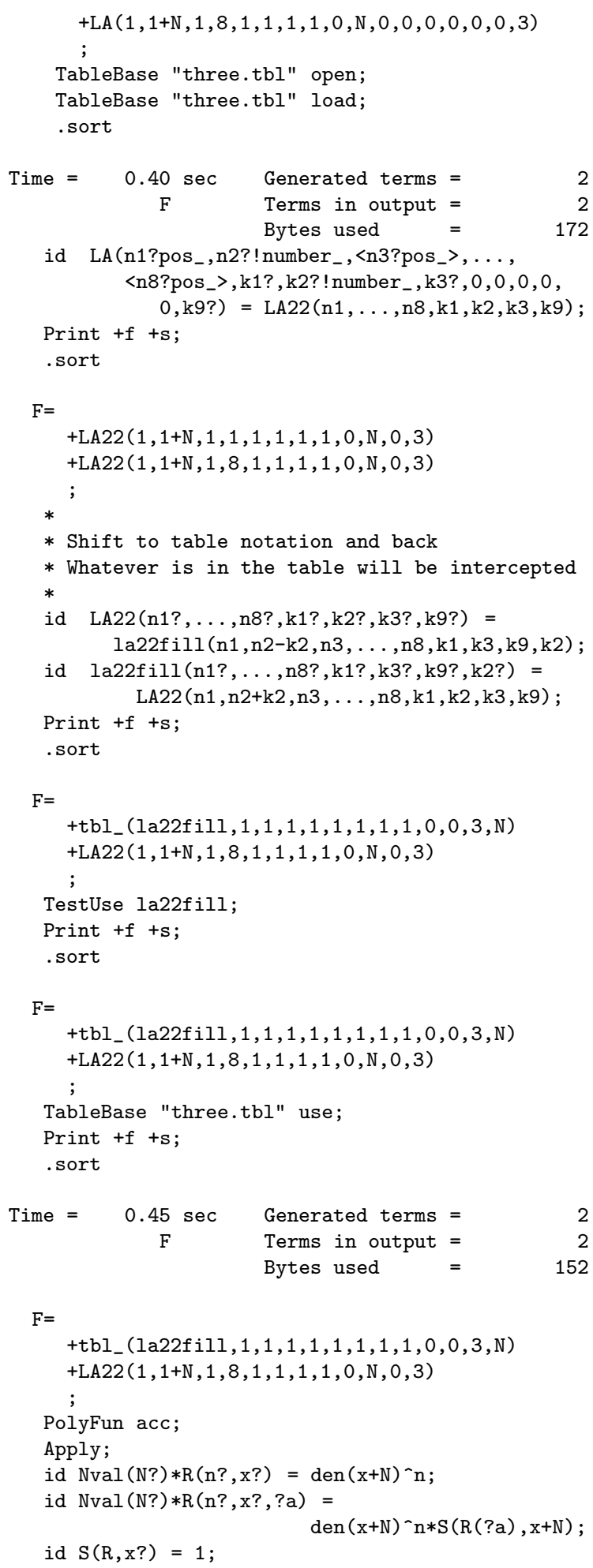




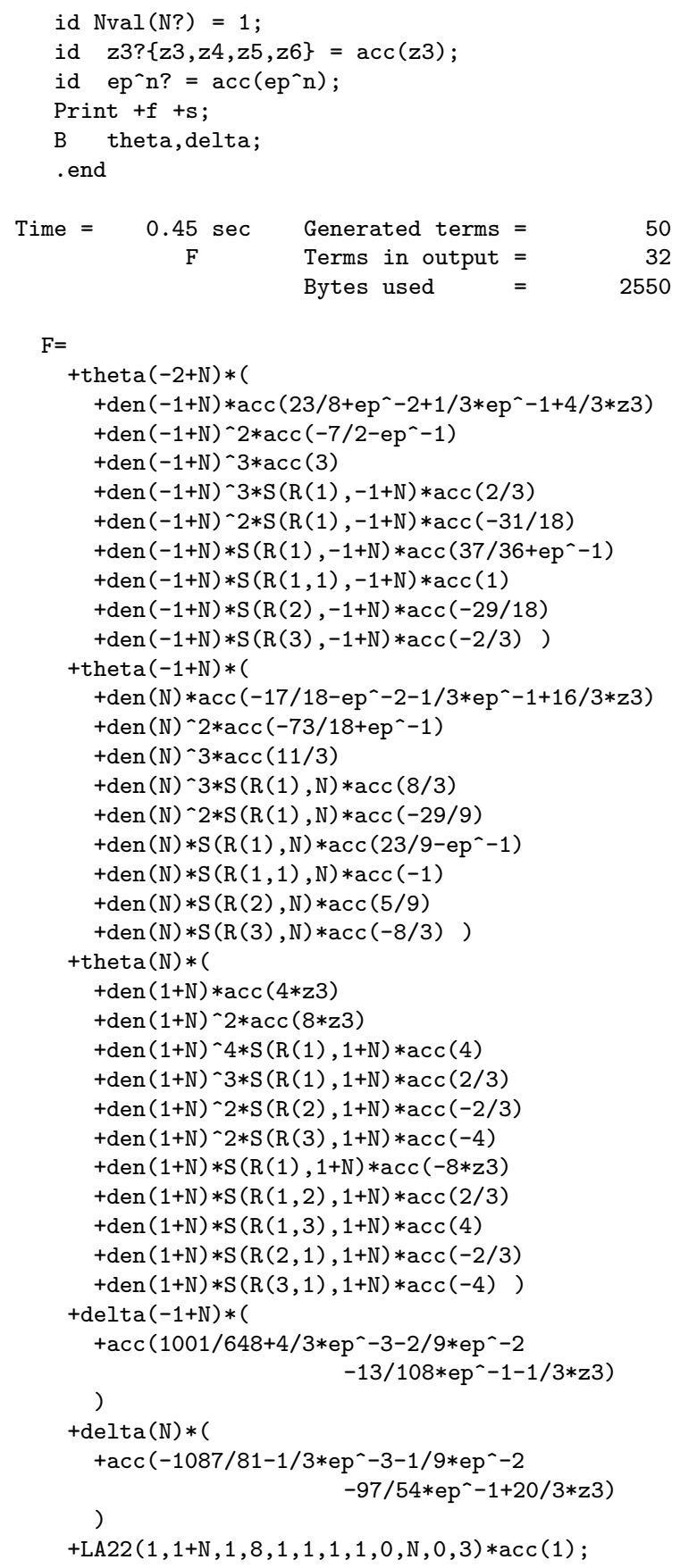

\section{Some extra remarks}

The above features are released in version 3.1 of FORM at its regular address http://www.nikhef.nl/ form.
Of course there are still features that FORM does not have and would be much appreciated. One would be proper GCD and factorization algorithms. This would make it much easier to solve sets of equations. These are anticipated, but lack of manpower is the main problem.

It seems that the inherent speed of FORM comes from its internal data representation. The locality of its operations seems to be less important in this matter than was previously believed. This plays mainly a role when expressions are so big that they reside on disk. But even in that case a good use of the .sort instructions helps.

Currently a study is under way to see whether FORM can be made into an open source project. This would need a considerable amount of manpower, because the sources may have to be reprogrammed, several levels of documentation will have to be made and a number of additions will have to made.

\section{REFERENCES}

[1] J. A. M. Vermaseren, math-ph/0010025

[2] Beffa G. Marí, J. A. Sanders and Jing Ping Wang, "Integrable systems in threedimensional Riemannian geometry", J. Nonlinear Sci., 12 (2002) 143-167

[3] Jan A. Sanders and Jing Ping Wang, "Combining Maple and Form to decide on integrability questions" Comput. Phys. Comm. 115 (1998) 447-459

[4] J. A. M. Vermaseren, Int. J. Mod. Phys. A14 (1999) 2037

[5] S. Moch, J. A. M. Vermaseren and A. Vogt hep-ph/0209100

[6] J. A. M. Vermaseren, S. Moch and A. Vogt, hep-ph/0211296

[7] D. Fliegner, A. Retey, and J. A. M. Vermaseren hep-ph/9906426,

[8] R. Kreckel, PhD thesis "Algorithmische Methoden zur Berechnung von Vierbeinfunktionen", Mainz 2002

[9] Gzip was written by Jean-loup Gailly and Mark Adler. Here we use version 1.1.3 